\title{
Delivery of gefitinib with an immunostimulatory nanocarrier improves therapeutic efficacy in lung cancer
}

\author{
Dingwei Diao ${ }^{1 \#}$, Jianxue Zhai ${ }^{1 \#}$, Jianjun Yang ${ }^{1 \#}$, Hua $\mathrm{Wu}^{1}$, Jianjun Jiang ${ }^{1}$, Xiaoying Dong ${ }^{1}$, \\ Antonio Passaro ${ }^{2}$, Beatrice Aramini ${ }^{3}$, Shuan Rao ${ }^{1 \wedge}$, Kaican Cai ${ }^{1}$ \\ ${ }^{1}$ Department of Thoracic Surgery, Nanfang Hospital, Southern Medical University, Guangzhou, China; ${ }^{2}$ Division of Thoracic Oncology, European \\ Institute of Oncology, Milan, Italy; ${ }^{3}$ Division of Thoracic Surgery, Department of Medical and Surgical Sciences, University of Modena and Reggio \\ Emilia, Modena, Italy \\ Contributions: (I) Conception and design: K Cai, S Rao; (II) Administrative support: H Wu, J Jiang, X Dong; (III) Provision of study materials or \\ patients: D Diao, J Zhai, J Yang; (IV) Collection and assembly of data: D Diao, J Zhai, J Yang; (V) Data analysis and interpretation: D Diao, J Zhai, J \\ Yang, A Passaro, B Aramini; (VI) Manuscript writing: All authors; (VII) Final approval of manuscript: All authors. \\ \#These authors contributed equally to this work. \\ Correspondence to: Kaican Cai; Shuan Rao. Department of Thoracic Surgery, Nanfang Hospital, Southern Medical University, no. 1838 Guangzhou \\ Avenue North, Baiyun District, Guangzhou 510515, China. Email: caican@smu.edu.cn; raoshuan1@smu.edu.cn.
}

Background: Combining different cancer treatments represents a promising strategy to improve the therapeutic outcome for lung cancer patients with or without druggable gene alterations.

Methods: We previously developed a polyethylene glycol-based (PEG-based) immunostimulatory nanocarrier ( $\mathrm{PEG}_{2 \mathrm{k}}$-Fmoc-NLG919) which can efficiently co-deliver an indoleamine 2,3-dioxygenase-1 (IDO1) inhibitor and the chemotherapeutic agent, paclitaxel. This method was found to improve cancer therapy by simultaneously performing immuno- and chemo-therapy. However, whether this nanocarrier could deliver targeted drugs to implement targeted therapy together with immunotherapy remains unclear.

Results: Here, we report that the delivery of the classical tyrosine kinase inhibitor (TKI), gefitinib, with the optimized PEG $_{5 \mathrm{k}}$-Fmoc-NLG919 nanocarrier, increased the sensitivity of lung cancer cells to gefitinib in vitro. Gefitinib was gradually but sufficiently released from the nanocarrier with comparable capacity to inhibit epidermal growth factor receptor (EGFR) activity as using free gefitinib directly. More importantly, treatment with gefitinib-loaded $\mathrm{PEG}_{5 \mathrm{k}}$-Fmoc-NLG919 could suppress lung tumor development more efficiently than gefitinib alone in vivo by inducing an immune active microenvironment with more functional $\mathrm{CD}^{+} \mathrm{T}$ cells and less regulatory $\mathrm{T}$ cell infiltration.

Conclusions: Our study therefore demonstrates that delivery of small molecular targeted drugs with the immunostimulatory nanocarrier is a straightforward strategy for improving antitumor response for lung cancer therapy.

Keywords: Gefitinib; nanocarrier; immunostimulatory; lung cancer

Submitted Nov 06, 2020. Accepted for publication Feb 26, 2021.

doi: $10.21037 /$ tlcr-21-144

View this article at: http://dx.doi.org/10.21037/tlcr-21-144

\footnotetext{
^ ORCID: 0000-0002-5268-7006.
} 


\section{Introduction}

Although chemotherapy, targeted therapy, and immunotherapy have improved cancer patients' prognosis in last few decades, lung cancer remains the leading cause of cancer-related death with the highest incidence among all cancer subtypes worldwide, with estimated 2,093,000 new and 1,761,000 death cases in 2018 (1). The classical platinum-based chemotherapy has been used for treating lung cancer patients for over 40 years, but the high toxicity, drug resistance, and limited therapeutic effect involved have hampered this approach as the primary selection for clinicians $(2,3)$. Targeted therapy predominantly using tyrosine kinase inhibitors (TKIs) has been proven to significantly improve lung cancer patients' prognosis, but is limited to only those patients who carry genetic mutations such as epidermal growth factor receptor (EGFR) or anaplastic lymphoma kinase (ALK). According to a research enrolled 1,770 patients with non-small cell lung cancer, there were $50.3 \%$ patients harbor EGFR mutation and only $4.3 \%$ patients carry ALK fusion (4). Even worse, drug resistance is usually quick to establish in these patients, and other treatments are inevitably required $(5,6)$. The newly emergent immunotherapy with immune checkpoint inhibitors, such as monoclonal antibody of programmed cell death protein 1 (PD-1), programmed cell death protein ligand 1 (PD-L1), and cytotoxic T lymphocyte-associated protein 4 (CTLA-4), can efficiently stimulate antitumor efficacy and thereby provide dramatic survival advantages for lung cancer patients $(7,8)$. The latest results from the randomized, phase III trials Checkmate 017 and 057 showed a 5 -year overall survival rate of $13.4 \%$ versus $2.6 \%$ for advanced non-small cell lung cancer patients who treated with nivolumab or docetaxel, respectively (9). However, immunotherapy is only effective in a small subpopulation of patients, and even these responsive patients may suffer severe side effects and subsequent cancer progression $(10,11)$. Therefore, the development of novel treatment methods and combination strategies for lung cancer patients is urgently needed.

Recently, accumulating evidence has suggested that combination of different therapies is a promising strategy for improving lung cancer patient prognosis. For instance, chemotherapy has been found to trigger antitumor immune responses (12) and enhance tumor cell susceptibility to cytotoxic T lymphocyte (CTL)-mediated killing during cancer immunotherapy, which then substantially increases the response to immunotherapy (13). Ipilimumab, one of the antibodies that specifically blocks binding between CTLA4 and its ligands (CD80/CD86) and subsequently augments T-cell activation and proliferation, has been shown to be recruited in many clinical immunochemotherapy treatment studies (14-16). Furthermore, TKI combined with pemetrexed and carboplatin-based chemotherapy was demonstrated to significantly increase EGFR-mutated Japanese lung cancer patients' overall survival as compared with targeted therapy using TKIs alone (17). However, no clear and solid clinical evidence has shown that combined targeted therapy with immunotherapy can bring substantive benefits for lung cancer patients so far, although some notable clinical trials have been launched to investigate this issue, including LUX-Lung IO, CheckMate 012, CheckMate 370, KEYNOTE-021 $(18,19)$.

Previously, we developed a polyethylene glycol-based (PEG-based) immunostimulatory nanocarrier $\left(\mathrm{PEG}_{2 \mathrm{k}}{ }^{-}\right.$ Fmoc-NLG919) for co-delivery of an indoleamine 2,3-dioxygenase-1 (IDO1) inhibitor (NLG919) and a chemotherapeutic agent, paclitaxel (PTX) (20). IDO1 also plays a vital role in establishing the immunosuppressive tumor microenvironment, however, the IDO1 inhibitor was poorly soluble under physiological conditions, while $\mathrm{PEG}_{2 \mathrm{k}}$ was used to dramatically increase the delivery efficiency. The Fmoc group was introduced to the carrier to increase the drug-loading capacity and formulation stability (21), and the nanocarrier $\mathrm{PEG}_{2 \mathrm{k}}$ was improved to $\mathrm{PEG}_{5 \mathrm{k}}$ for better delivery efficiency (22). The systemic delivery of PTX using this nanocarrier could induce significantly increased antitumor response in breast cancer and melanoma murine models (20); however, whether the delivery of TKIs using the $\mathrm{PEG}_{5 \mathrm{k}}-\mathrm{Fmoc}-\mathrm{NLG} 919$ can provoke synergistic antitumor effect remains unclear. In the present study, we aimed to explore whether the delivery of gefitinib using PEG $_{5 \mathrm{k}}$-Fmoc-NLG919 and gefitinib could provide improved prognosis for lung cancer with both in and ex vivo lung cancer models.

We present the following article in accordance with the ARRIVE reporting checklist (available at http://dx.doi. org/10.21037/tlcr-21-144).

\section{Methods}

\section{Chemical materials}

Gefitinib was purchased from LC labs (MA, USA), while RPMI 1640 medium, fetal bovine serum (FBS), and penicillin-streptomycin solution $(100 \times)$ were all purchased 


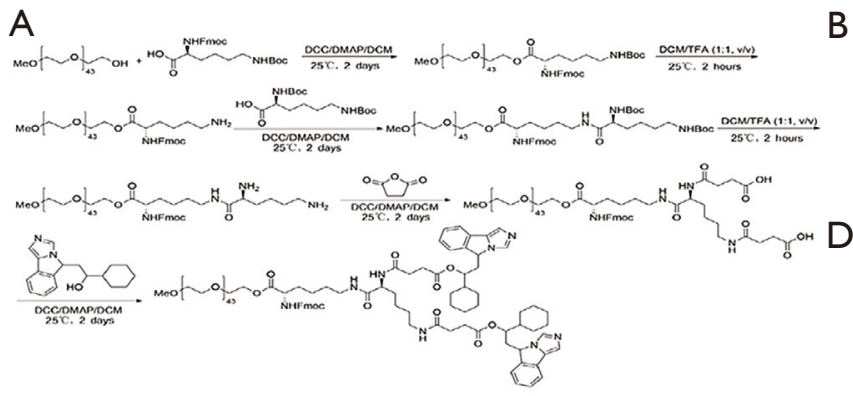

B
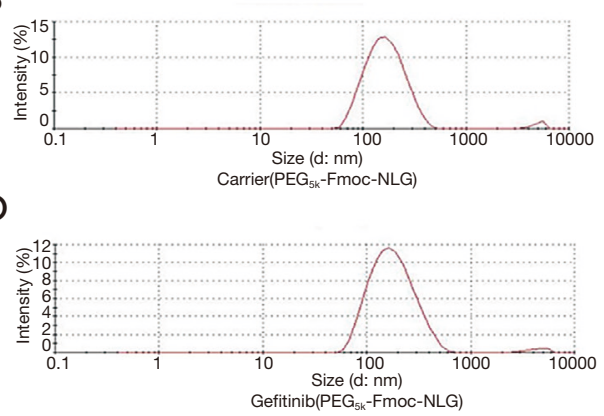

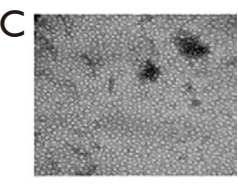

$\mathrm{E}$

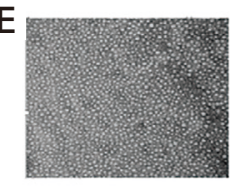

Figure 1 In vitro synthesis and characterizations of gefitinib/PEG ${ }_{5 \mathrm{k}}-\mathrm{Fmoc}_{\mathrm{N}} \mathrm{NLG} 919$ micelles. (A) Synthesis scheme of gefitinib/PEG ${ }_{5 \mathrm{k}}-$ Fmoc-NLG919. (B) Size distribution of drug-free PEG ${ }_{5 \mathrm{k}}-$ Fmoc-NLG919 micelles were examined by dynamic light. (C) Morphology of drug-free PEG $_{5 \mathrm{k}}$-Fmoc-NLG919 micelles were examined by TEM. (D) Size distributions of gefitinib-loaded PEG ${ }_{5 \mathrm{k}}-\mathrm{Fmoc}_{\mathrm{N}}-\mathrm{NLG}$ 919 micelles (carrier: drug, 2.5:1, m/m) were examined by dynamic light scattering. (E) Morphologies of gefitinib-loaded PEG ${ }_{5 \mathrm{k}}$-Fmoc-NLG919 micelles (carrier:drug, 2.5:1, m/m) was examined by TEM. TEM, transmission electron microscope.

from Invitrogen (NY, USA). Monomethoxy $\mathrm{PEG}_{5 \mathrm{~K}}$ and 4-dimethylaminopyridine (DMAP) were obtained from Sigma Aldrich (Germany).

\section{Animals}

C57BL/6 mice (6-8 weeks) were purchased from Charles River (CA). All animals were housed under pathogenfree conditions according to Association for Assessment and Accreditation of Laboratory Animal Care (AAALAC) guidelines. Experiments were performed under a project license (NO.: NFEC-2020-094) granted by institutional ethics board of Nanfang Hospital, Southern Medical University, in compliance with Chinese national or institutional guidelines for the care and use of animals.

\section{Cell culture}

Lewis lung carcinoma (3LL) murine lung cancer cells and A549 human lung cancer cells were maintained in RPMI 1640 medium supplemented with $10 \%$ FBS and $1 \%$ penicillin-streptomycin at $37{ }^{\circ} \mathrm{C}$ in a humidified environment with $5 \% \mathrm{CO}_{2}$. All cell lines used in this work were obtained from American Type Culture Collection (ATCC, VA, USA).

\section{Synthesis of PEG ${ }_{5 k}$-Fmoc-NLG919 conjugate}

PEG $_{5 \mathrm{k}}-$ Fmoc-NLG919 conjugate was synthesized and purified following a published protocol and is outlined in Figure 1 (22).

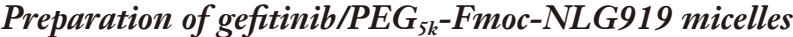

Gefitinib-loaded PEG $_{5 \mathrm{k}}$-Fmoc-NLG919 micelles were prepared via a film-hydration method. Gefitinib $[2.5 \mathrm{mmol}$ in dehydrated culture media (DCM)] and $\mathrm{PEG}_{5 \mathrm{k}}-\mathrm{Fmoc}-$ NLG919 (10 mmol) were mixed at different ratios in glass tubes. Saline was added after removing the organic solvent. Other micelles were similarly prepared. The particle size of these micelles was measured by dynamic light scattering (DLS), and the morphologies were measured by transmission electron microscope (TEM).

\section{Gefitinib release assay}

The kinetics of gefitinib in vitro release from gefitinib/ $\mathrm{PEG}_{5 \mathrm{k}}-$ Fmoc-NLG2 was studied using a dialysis method. Briefly, $1 \mathrm{~mL}$ of gefitinib/PEG ${ }_{5 \mathrm{k}}$-Fmoc-NLG2 micelles containing $1 \mathrm{mg}$ of gefitinib were placed in a clamped dialysis bag (MWCO $3.5 \mathrm{kDa}$ ) and immersed in $25 \mathrm{~mL}$ of phosphate-buffered saline (PBS) solution containing $0.5 \%(\mathrm{w} / \mathrm{v})$ Tween. The experiment was performed in an incubation shaker at $37^{\circ} \mathrm{C}$ at $100 \mathrm{rpm}$. At selected time intervals, both $10 \mu \mathrm{L}$ of gefitinib/PEG ${ }_{5 \mathrm{k}}-\mathrm{Fmoc}-\mathrm{NLG} 2$ micelle solution in the dialysis bag and $1 \mathrm{~mL}$ of medium outside the dialysis bag were withdrawn while the same amount of fresh medium was added for replenishment. For comparison, free gefitinib dissolved in $2 \%$ dimethyl sulfoxide (DMSO) was included as free diffusion control. The gefitinib released from micelles was also measured by Waters e2695 HPLC system equipped with a Waters 2489 UV detector (MA, USA). Then, $100 \mathrm{mM}$ of ammonium 
acetate $(\mathrm{pH}=5)$ and acetonitrile $(60: 40, \mathrm{v} / \mathrm{v})$ was used for the mobile phase. Gefitinib was detected at a $248 \mathrm{~nm}$ wavelength.

\section{In vitro MTT assay}

A549 and 3LL lung cancer cell lines $\left(5 \times 10^{3}\right.$ cells/well) were seeded in 96-well plates and incubated for 24 hours. Cells were then treated with carrier alone, gefitinib, or gefitinib/ $\mathrm{PEG}_{5 \mathrm{k}}$-Fmoc-NLG919 in different concentrations. The cell viabilities were determined by MTT assay.

\section{Western blotting}

A549 or 3 LL lung cancer cell lines $\left(7 \times 10^{3}\right.$ cells/well $)$ were seeded in 6-well plates and incubated for 24 hours. Cells were then treated with blank medium, carrier alone, gefitinib, or gefitinib/PEG sk $_{5 \mathrm{k}}$-Fmoc-NLG919 for 48 hours. Cells and the tumors after homogenization were incubated with radioimmunoprecipitation assay buffer (RIPA) in a $4{ }^{\circ} \mathrm{C}$ room for 1 hour. These samples were centrifuged at 12,500 rpm for 10 minutes, and the precipitation was discarded. Loading buffer with one-fourth of the supernatant volume was added, and the samples were heated at $95{ }^{\circ} \mathrm{C}$ for 5 minutes. The expression of EGFR and p-EGFR in these total protein samples were evaluated by western blotting. The EGFR [4267] and p-EGFR antibody [2235] used were acquired from Cell Signal Technology, Inc.

\section{In vivo antitumor activity}

To investigate antitumor activity of gefitinib-loaded $\mathrm{PEG}_{5 \mathrm{k}}{ }^{-}$ Fmoc-NLG919 micelles, C57BL/6 mice were inoculated with 3LL tumor cells on the right flank area, and randomly divided into four groups $(n=4)$ : control, blank carrier, free gefitinib, and gefitinib/PEG ${ }_{5 \mathrm{k}}$-Fmoc-NLG919 (with a molar ratio of 1:10). Mice were treated with various drugs after the tumor volume reached about $50 \mathrm{~mm}^{3}$, once every 3 days 5 times through tail vein injection (dosage of gefitinib: $10 \mathrm{mg} / \mathrm{kg}$ ). The tumor volume and body weighs of mice were monitored. Tumor sizes were measured with the digital caliper every 3 days following the initiation of the treatment and calculated by the following formula: ( $\mathrm{L} \times$ $\left.\mathrm{W}^{2}\right) / 2$, where $\mathrm{L}$ is the longest diameter and $\mathrm{W}$ is the shortest diameter of tumor (mm). At the end of the experiment, tumors were collected for hematoxylin and eosin (HE) and immunohistochemical (anti-Ki67) staining analysis.

\section{Quantification of tumor-infiltrating lymphocytes}

Tumor-bearing mice were intravenously administered with various agents once every 3 days 3 times. Tumor tissues were harvested at 24 hours after the final treatment, and then the single cell suspension was collected and stained with various antibodies (CD8, CD4, granzyme B, IFN- $\gamma$, and PD-1) for fluorescence-activated cell sorting (FACS) evaluation.

The immune cell populations in the tumor tissues were analyzed by flow cytometry. Cell suspensions from tumor tissues were filtered, and red blood cells were lysed. For extracellular staining, cells were incubated with the indicated combinations of antibodies (CD8, CD4, and CD45). For intracellular staining, cells were fixed and permeabilized immediately after cell surface staining according to the manufacturer's instructions. Briefly, fixation buffer was diluted 4 times, and incubated with cell for $20 \mathrm{mins}$. Then, combinations of antibodies (FoxP3, IFN- $\gamma$, and granzyme B) were added to cells in permeabilization buffer. For IFN- $\gamma$ and granzyme B staining, cells were stimulated with phosphomolybdic acid (PMA, $5 \mathrm{ng} / \mathrm{mL}$ ) and ionomycin $(500 \mathrm{ng} / \mathrm{mL})$ in the presence of $10 \mu \mathrm{g} / \mathrm{mL}$ brefeldin A (BFA) for 4 hours followed by extracellular and intracellular staining. All antibodies were purchased from BD Biosciences, and data were collected on an LSRFortessa (BD Biosciences). The data were analyzed by FlowJo software.

\section{Statistical analysis}

All data are presented as mean \pm standard error of the mean (SEM). Differences between groups were assessed using ANOVA, and a $\mathrm{P}$ value $<0.05$ was considered statistically significant.

\section{Results}

\section{Characterization of blank and gefitinib/PEG $G_{5 k}-F m o c-$ NLG919 micelles}

The blank and gefitinib-loaded PEG $_{5 \mathrm{k}}$-Fmoc-NLG919 micelles were prepared using a film hydration method (Figure 1A). The critical micelle concentration (CMC), particle size, and morphologies of PEG $_{5 \mathrm{k}}-$ Fmoc-NLG919 micelles were measured according to a previously described method (20). The average size of PEG $_{5 \mathrm{k}}$-Fmoc-NLG919 and gefitinib/PEG ${ }_{5 \mathrm{k}}-$ Fmoc-NLG919 as revealed by the DLS had a major peak of 175.5 and $191.8 \mathrm{~nm}$, respectively (Figure 1B,C). The morphology of micelles with and 


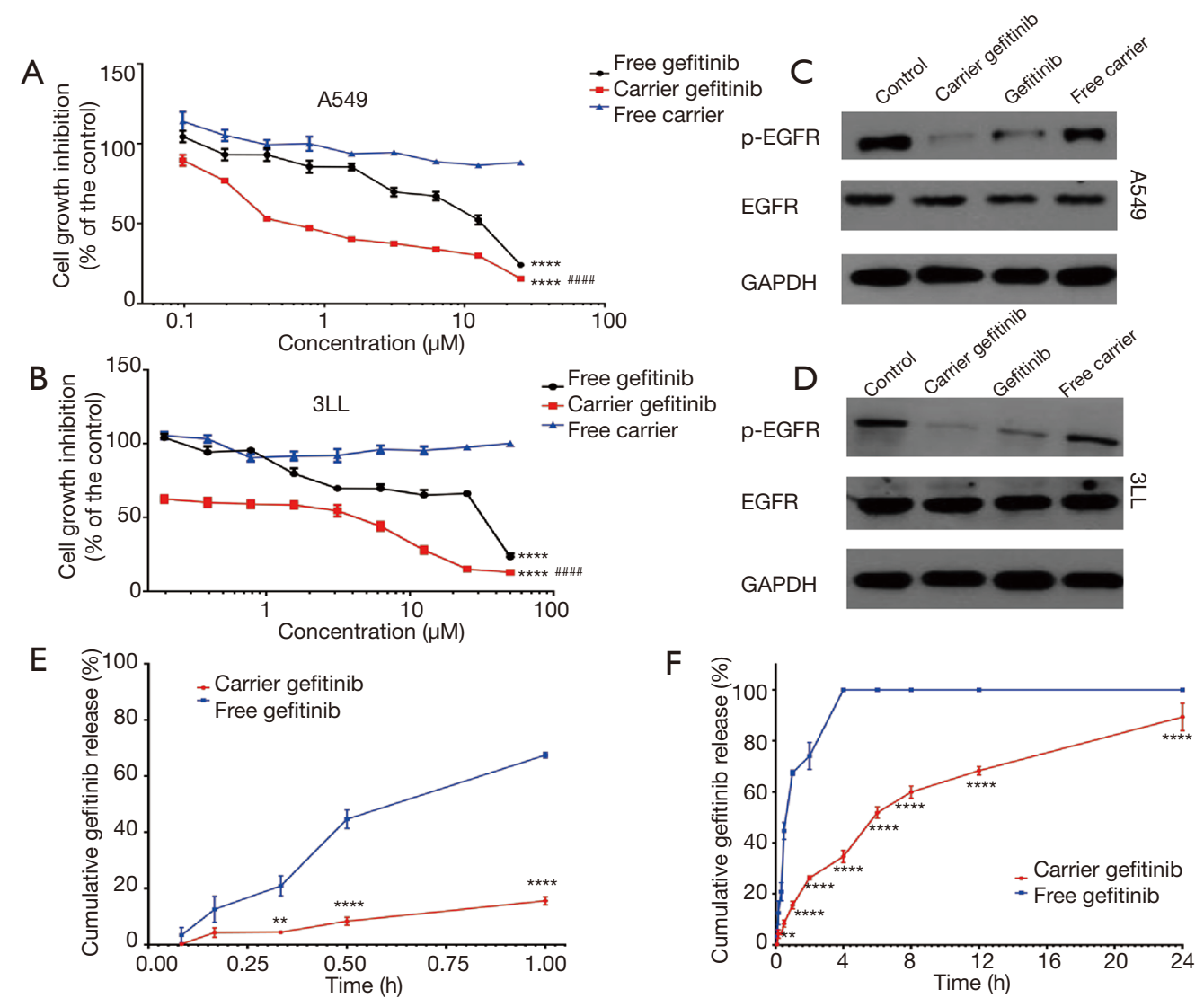

Figure 2 In vitro evaluation of anti-lung cancer efficacy of gefitinib-loaded PEG ${ }_{5 \mathrm{k}}$-Fmoc-NLG919. (A) Cytotoxicity analysis PEG F $_{5 \mathrm{k}}$-FmocNLG919 (carrier) alone, free gefitinib, and gefitinib/PEG ${ }_{5 \mathrm{k}}$-Fmoc-NLG919 (carrier gefitinib) against A549 human lung cancer cell line. ****, $\mathrm{P}<0.0001$ (free gefitinib, carrier gefitinib vs. free carrier group); ${ }^{\# \# \#, ~} \mathrm{P}<0.0001$ (carrier gefitinib vs. gefitinib group). (B) Cytotoxicity evaluation of $\mathrm{PEG}_{5 \mathrm{k}}-$ Fmoc-NLG919 alone, free gefitinib, and gefitinib/PEG ${ }_{5 \mathrm{k}}$-Fmoc-NLG919 in 3LL mouse lung cancer cell line (3LL).

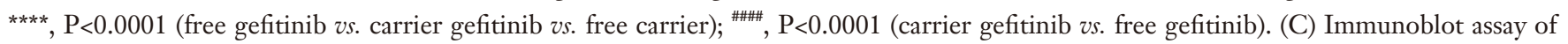
EGFR activity in A549 cells treated with PEG $_{5 \mathrm{k}}-$ Fmoc-NLG919 alone, free gefitinib, and gefitinib/PEG ${ }_{5 \mathrm{k}}$-Fmoc-NLG919, with GAPDH used as the loading control. (D) Western blot analysis of EGFR activity in 3LL cells treated with PEG 5 -Fmoc-NLG919 alone, free gefitinib, and gefitinib/PEG ${ }_{5 \mathrm{k}}-$ Fmoc-NLG919. (E) The 1-hour drug releasing assay of gefitinib/PEG 5 -Fmoc-NLG919, with free gefitinib used as the control. **, $\mathrm{P}<0.01$; ${ }^{* * *}, \mathrm{P}<0.0001$. (F) The 24-hour drug releasing assay of gefitinib/PEG ${ }_{5 \mathrm{k}}-\mathrm{Fmoc} \mathrm{NLG}$ 919, with free gefitinib used as the control. **, $\mathrm{P}<0.01 ;{ }^{* * * *}, \mathrm{P}<0.0001$.

without gefitinib-loaded PEG $_{5 \mathrm{k}}$-Fmoc-NLG919 analyzed by TEM showed that both the carrier and gefitinib/ $\mathrm{PEG}_{5 \mathrm{k}}-$ Fmoc-NLG919 produced an even-size spherical morphology (Figure 1D,E).

\section{In vitro cytotoxicity evaluation of gefitinib/PEG $G_{5 k}-F m o c-$ NLG919 micelles}

The cytotoxicity of blank carrier and gefitinib-loaded micelles was examined by the MTT assay. The carrier alone showed very little cytotoxicity in both A549 and 3LL cell lines (Figure $2 A, B$ ). The $\mathrm{IC}_{50}$ of gefitinib/PEG ${ }_{5 \mathrm{k}}-\mathrm{Fmoc}-$ NLG919 and free gefitinib were 1.107 and $10.53 \mu \mathrm{M}$ in the A549 cell line, respectively (Figure $2 A$ ); similarly, the $\mathrm{IC}_{50}$ value of gefitinib/PEG ${ }_{5 \mathrm{k}}-$ Fmoc-NLG919 was $1.826 \mu \mathrm{M}$, as compared to that of $23.67 \mu \mathrm{M}$ of gefitinib alone in murine lung cancer 3LL cells (Figure 2B); this suggested a significant increase of the killing effect in lung cancer cells for gefitinib when delivered with $\mathrm{PEG}_{5 \mathrm{k}}-\mathrm{Fmoc}-$ NLG919. Furthermore, we compared the EGFR activity in both A549 and 3LL cells which were treated with carrier, gefitinib, and gefitinib/PEG ${ }_{5 \mathrm{k}}$-Fmoc-NLG919. In line with 
the cytotoxicity assay, when delivered with $\mathrm{PEG}_{5 \mathrm{k}}-\mathrm{Fmoc}$ NLG919, gefitinib showed better efficiency in suppressing EGFR activity (Figure 2C,D). These data collectively indicated that the use of $\mathrm{PEG}_{5 \mathrm{k}}$-Fmoc-NLG919 micelles was a powerful system to wrap and deliver small molecular drugs, such as gefitinib.

\section{Gefitinib was gradually but sufficiently released from the gefitinib/PEG ${ }_{5 k}-$ Fmoc-NLG919 micelles}

To evaluate how gefitinib is released from gefitinib/PEG $\mathrm{P}_{5 \mathrm{k}}{ }^{-}$ Fmoc-NLG919, we performed a dialysis assay to determine the kinetics of gefitinib's release in a time-dependent fashion. A 1-hour short-period experiment indicated that roughly $20 \%$ of the gefitinib could be gradually released from the gefitinib/PEG ${ }_{5 \mathrm{k}}$-Fmoc-NLG919, which was dramatically slower than the free-gefitinib release (Figure 2E). Similarly, in a 24-hour period assay, we also observed that gefitinib could be slowly unloaded from the micelles as compared to free gefitinib, but about $90 \%$ of the gefitinib was released from the micelles after 24 hours, suggesting that $\mathrm{PEG}_{5 \mathrm{k}}-\mathrm{Fmoc}-\mathrm{NLG} 919$ was a robust vector to satisfactorily release the small molecular drugs (Figure 2F).

\section{Gefitinib/PEG $G_{5 k}-$ Fmoc-NLG919 micelles show improved antitumor effect over gefitinib alone}

In order to evaluate the in vivo antitumor effect of carrier loaded gefitinib, we established a mouse lung cancer xenograft model using 3LL cells. Mice bearing 3LL tumors received different treatments via tail vein injection once every 3 days for a total of 3 times. Compared to the control group mice which received saline injection, mice treated with PEG $_{5 \mathrm{k}}$-Fmoc-NLG919 did not show any antitumor effects while the mice treated with gefitinib alone only showed mild effects in delaying tumor progression; however, when mice were treated with the gefitinib/PEG $\mathrm{Pk}_{5 \mathrm{k}}-\mathrm{Fmoc}$ NLG919 micelles, the tumor development was significantly inhibited as revealed by the tumor growth curve (Figure 3A). In line with this, tumor size evaluation suggested that only gefitinib/PEG ${ }_{5 \mathrm{k}}-$ Fmoc-NLG919 treatment could dramatically suppress the tumor formation as compared to all other treatments (Figure 3B). We then analyzed the EGFR activity in the tumors isolated from each group, and, not surprisingly, gefitinib treatment could efficiently block EGFR activity, while a comparable inhibition effect could be achieved by gefitinib/PEG ${ }_{5 \mathrm{k}}$-Fmoc-NLG919 treatment
(Figure 3C), further supporting $\mathrm{PEG}_{5 \mathrm{k}}-$ Fmoc-NLG919 micelles as an efficient system to deliver gefitinib both ex vivo and in vivo. Moreover, histology examination indicated that tumors treated with gefitinib/ $\mathrm{PEG}_{5 \mathrm{k}}$-FmocNLG919 consistently exhibited markedly increased apoptotic figures or necrotic areas (Figure 3D), while proliferation of lung tumors as detected by Ki67 staining also suggested that gefitinib/PEG ${ }_{5 \mathrm{k}}-\mathrm{Fmoc}-\mathrm{NLG} 919$ could substantially suppress lung tumor cell proliferation (Figure 3E).

\section{Gefitinib/PEG $G_{5 k}-$ Fmoc-NLG919 micelles reshaped immune cell compositions in the tumor microenvironment}

Since the $\mathrm{PEG}_{5 \mathrm{k}}$-Fmoc-NLG919 nanocarrier harbors an IDO1 inhibitor (NLG919), we then examined whether gefitinib/PEG ${ }_{5 \mathrm{k}}$-Fmoc-NLG919 micelles could affect the immune cell infiltration into the tumors. Tumor tissues from mice with different treatments were collected and digested for immune cell population assay using flow cytometry. Intriguingly, treatment with gefitinib/ $\mathrm{PEG}_{5 \mathrm{k}^{-}}$ Fmoc-NLG919 resulted in the highest infiltration of functional granzyme B-positive $\mathrm{CD}^{+} \mathrm{T}$ cells in the tumor microenvironment (Figure 4A,B); in contrast, the infiltration of regulatory $\mathrm{T}$ cells (Treg) which were identified as $\mathrm{CD}^{+}$ and $\mathrm{FoxP}_{3}{ }^{+}$were significantly decreased in the gefitinib/ $\mathrm{PEG}_{5 \mathrm{k}}$-Fmoc-NLG919-treated tumors (Figure 4C,D), suggesting that gefitinib/PEG ${ }_{5 \mathrm{k}}-\mathrm{Fmoc}-\mathrm{NLG} 919$ could not only inhibit EGFR activity as free gefitinib in vivo, but could also synergistically stimulate the local antitumor immune response via inhibiting IDO activity.

\section{Discussion}

Increasing evidence has shown that combining different cancer therapies is a promising strategy to improve lung cancer patients' prognosis (23-26). For instance, it has been previously reported that TKI could augment the expression of MHC classes I and II genes and thus promote the antigen presentation (27); besides, TKI could enhance the production of interferon- $\gamma$ and inhibit the apoptosis of $\mathrm{T}$ cells in the tumor microenvironment (12), indicating combination of targeted and immunotherapy might improve therapeutic efficacy for lung cancer patients, however, it is still unclear whether such combination strategy could induce severe adverse effect. Our previously developed PEGNLG-based immunostimulatory nanocarrier $\left(\mathrm{PEG}_{2 \mathrm{k}}-\mathrm{Fmoc}-\right.$ NLG919) for simultaneously delivering IDO inhibitor 

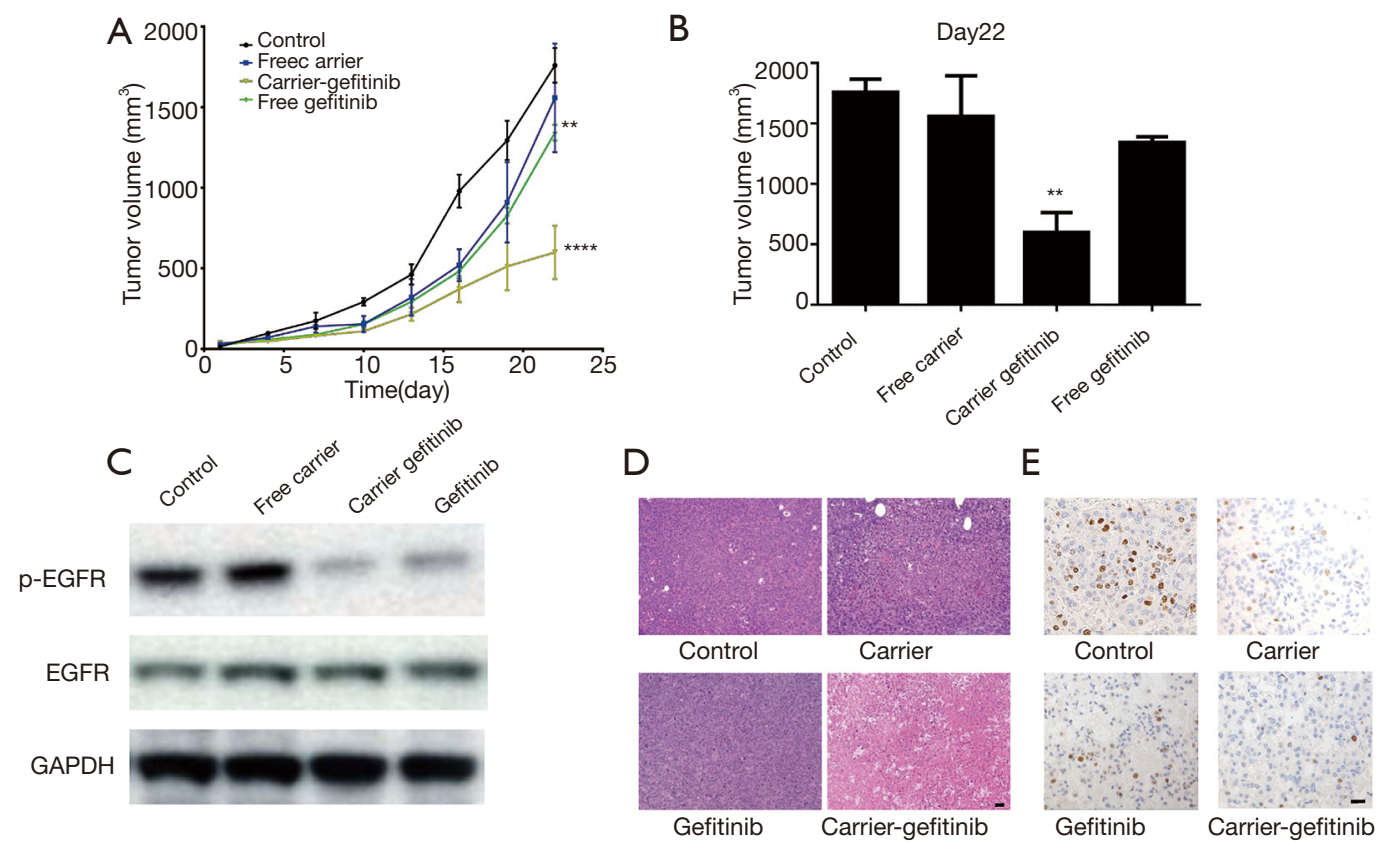

Figure 3 In vivo anti-lung cancer assay of gefitinib/PEG ${ }_{5 \mathrm{k}}-\mathrm{Fmoc}-\mathrm{NLG} 919$. (A) Tumor growth curve of tumor-bearing mice treated with PBS, PEG ${ }_{5 \mathrm{k}}$-Fmoc-NLG919 alone, free gefitinib, and gefitinib/PEG ${ }_{5 \mathrm{k}}$-Fmoc-NLG919. **, $\mathrm{P}<0.01$ (free gefitinib vs, control); ****, $<<0.0001$ (carrier-gefitinib vs. control). (B) Tumor volume evaluation of tumor-bearing mice on the day 22 after treating with $\mathrm{PBS}_{\mathrm{B}} \mathrm{PEG}_{5 \mathrm{k}}-\mathrm{Fmoc}_{\mathrm{m}}$ NLG919 alone, free gefitinib, and gefitinib/PEG ${ }_{5 \mathrm{k}}-\mathrm{Fmoc}_{\mathrm{N}} \mathrm{NG} 919 .{ }^{* *}, \mathrm{P}<0.01$ (carrier-gefitinib vs. free gefitinib). (C) Immunoblot assay of EGFR activity in the tumor tissues isolated from the mice treated with PEG $_{5 \mathrm{k}}-$ Fmoc-NLG919 alone, free gefitinib, and gefitinib/PEG ${ }_{5 \mathrm{k}}{ }^{-}$

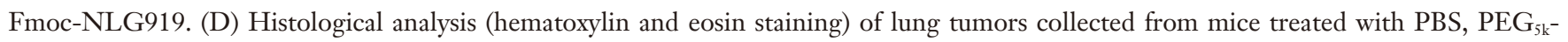
Fmoc-NLG919 alone, free gefitinib, and gefitinib/PEG ${ }_{5 \mathrm{k}}-$ Fmoc-NLG919; scale bars: $100 \mu \mathrm{m}$. (E) Proliferation assay (Ki67 staining) of lung tumors isolated from mice treated with PBS, PEG ${ }_{5 \mathrm{k}}-$ Fmoc-NLG919 alone, free gefitinib, and gefitinib/PEG ${ }_{5 \mathrm{k}}-\mathrm{Fmoc}_{-\mathrm{NLG}}$ 919; scale bars: $50 \mu \mathrm{m}$.

(NLG919) and a chemotherapeutic agent, PTX, has shown great advantage in stimulating antitumor response in breast cancer and melanoma murine models (20). Unlike many other drug carriers which are "inert", the PEG-Fmoc-NLG is a pro-drug that exhibits immunostimulatory activity by suppressing IDO activity. This property can induce additional tumor-suppressing effects by not only targeting the tumor cells directly, but also by modulating local immune response in the tumor microenvironment. Indeed, as indicated by the immunological analysis, there were increased functional $\mathrm{CD} 4^{+}$and $\mathrm{CD} 8^{+} \mathrm{T}$ cells, but decreased Treg and myeloid-derived suppressor cells infiltrated in the tumor tissues of mice treated with $\mathrm{PEG}_{2 \mathrm{k}}$-Fmoc-NLG919, compared with vehicle treated mice $(20,22)$.

Some studies have investigated the efficacy of combination of anti PD-1/PD-L1-based immunotherapy and targeted therapy in clinic; unfortunately, according to recent reports, the efficacy of such strategies is limited by severe adverse effects $(19,28,29)$. Thus, whether combining targeted and immune therapy is still a feasible method to achieve better antitumor performance needs to be urgently resolved. Importantly, it should be noted that IDO, in addition to PD-1/PD-L1/CTLA-4, plays a vital role in establishing the immunosuppressive microenvironment (30-32). To our knowledge, there are no ongoing clinical studies that are aimed at exploring the potential therapeutic effect of using IDO inhibitor in addition to small molecular targeted drugs.

In our study, we used the optimized $\mathrm{PEG}_{5 \mathrm{k}}-\mathrm{Fmoc}-$ NLG919 micelles as the carrier for loading the classical EGFR inhibitor, gefitinib to treat lung cancer. Surprisingly, the $\mathrm{IC}_{50}$ evaluation showed that gefitinib/ $\mathrm{PEG}_{5 \mathrm{k}}-\mathrm{Fmoc}-$ NLG919 could kill lung tumor cells more efficiently than free gefitinib; furthermore, it was observed that the EGFR activity in gefitinib/ $\mathrm{PEG}_{5 \mathrm{k}}$-Fmoc-NLG919-treated cells was lower than that of free gefitinib-treated cells. This could be 

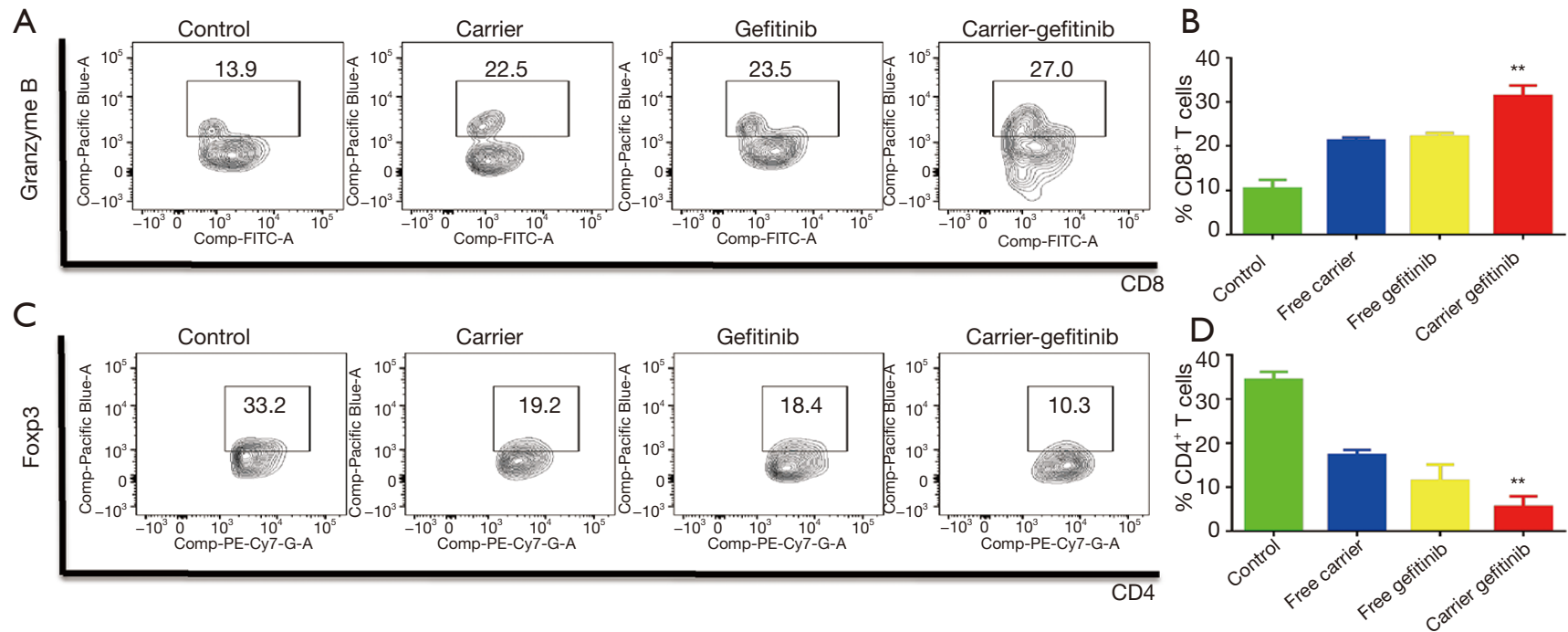

Figure 4 The tumor microenvironment evaluation after gefitinib/PEG ${ }_{5 \mathrm{k}}$-Fmoc-NLG919 treatment. (A,B) The FACs analysis of functional $\mathrm{CD}^{+}$cytotoxic $\mathrm{T}$ cell infiltration in the lung tumors treated with PBS, PEG $_{5 \mathrm{k}}-\mathrm{Fmoc}_{-\mathrm{NLG}}$ 919 alone, free gefitinib, and gefitinib/PEG ${ }_{5 \mathrm{k}^{-}}$ Fmoc-NLG919 (A) and the quantification (B). **, $\mathrm{P}<0.01$ (carrier-gefitinib vs. control). (C,D) The FACs analysis of regulatory T cell infiltration in the lung tumors treated with PBS, PEG ${ }_{5 \mathrm{k}}-$ Fmoc-NLG919 alone, free gefitinib, and gefitinib/PEG 5 -Fmoc-NLG919 (C) and the quantification (D). ${ }^{* *}, \mathrm{P}<0.01$ (carrier-gefitinib vs. control).

partially explained through use of the cumulative gefitinib releasing assay, which indicated that gefitinib was gradually and slowly released from the micelles, providing a constant proliferation stress for lung tumor cells. Moreover, the long-term cumulative releasing assay revealed that roughly $90 \%$ of the gefitinib could be released from the $\mathrm{PEG}_{5 \mathrm{k}}{ }^{-}$ Fmoc-NLG919 after 24 hours, suggesting that delivery of gefitinib with our nanocarrier did not impair the final dose compared to administering gefitinib directly.

Another major concern is whether the transport of gefitinib using PEG $_{5 \mathrm{k}}$-Fmoc-NLG919 could facilitate a synergistical anti-lung cancer effect in vivo. For this purpose, we optimized the nanocarrier $\mathrm{PEG}_{2 \mathrm{k}}$ to $\mathrm{PEG}_{5 \mathrm{k}}$ for better delivery efficiency (22); the Fmoc group was introduced to increase the drug-loading capacity and formulation stability (21). Additionally, the IDO inhibitor, NLG919, was also included, which endowed the carrier with immune-stimulating capacity. Using the classical lung cancer xenograft mouse model, we repeatedly confirmed that gefitinib/PEG ${ }_{5 \mathrm{k}}$-Fmoc-NLG919 exhibited better antitumor efficacy than gefitinib or carrier alone. By evaluating the immune cell subpopulations in the tumor microenvironment after different treatments, we demonstrated that gefitinib/PEG ${ }_{5 \mathrm{k}}-\mathrm{Fmoc}-\mathrm{NLG} 919$ could induce an immune-active microenvironment with more functional $\mathrm{CD} 8^{+} \mathrm{T}$ cells and less Treg infiltration. Thus, we concluded that delivery of gefitinib using our optimized PEG $_{5 \mathrm{k}}$-Fmoc-NLG919 could kill tumor cells by not only inhibiting EGFR activity directly, but also by rewiring the tumor microenvironment to form a more hostile surrounding that indirectly suppresses tumor cell proliferation. Of note, the slow-releasing capacity of gefitinib/PEG ${ }_{5 \mathrm{k}}-$ Fmoc-NLG919 might also contribute to its better antitumor performance by sustaining a continuous drug concentration in vivo. Nevertheless, the possible advantages of deliver gefitinib using PEG $_{5 \mathrm{k}}$-Fmoc-NLG919 micelles includes several aspects, first of all, the PEGFmoc-NLG is a pro-drug that exhibits immunostimulatory activity by suppressing IDO activity given that IDO plays a vital role in establishing the immunosuppressive microenvironment in tumor. Secondly, gefitinib could be gradually released from the micelles, providing a constant proliferation stress for lung tumor cells and reduce the $\mathrm{IC}_{50}$, finally, it could induce an immune-active microenvironment with more functional $\mathrm{CD} 8^{+} \mathrm{T}$ cells and less Treg infiltration.

Taken together, our study has demonstrated that delivering small molecular targeted drugs with our modified immunostimulatory micelles can achieve a synergistic and improved antitumor effect. Since gefitinib is a firstgeneration TKI, determining whether delivery of new 
generation TKIs with $\mathrm{PEG}_{5 \mathrm{k}}$-Fmoc-NLG919 could also provide prognostic advantage and whether this method could result in severe side effects, requires further investigation. Finally, we have proven that the concept of combining targeted and immune therapy is a promising strategy to improve the prognosis for cancer patients, and that using immunostimulatory nanocarriers to distribute targeted drugs provides an ideal approach for ultimately achieving simultaneous targeted and immune therapy.

\section{Acknowledgments}

We thank all colleagues from the Center for Pharmacogenetics, at Department of Pharmaceutical Sciences, School of Pharmacy, University of Pittsburgh for their kind support and help. The authors appreciate the academic support from AME Lung Cancer Collaborative Group.

Funding: This work was supported by Outstanding Youths Development Scheme of Nanfang Hospital, Southern Medical University (2020J001) and start-up funding from Southern Medical University.

\section{Footnote}

Reporting Checklist: The authors have completed the ARRIVE reporting checklist. Available at http://dx.doi. org/10.21037/tlcr-21-144

Data Sharing Statement: Available at http://dx.doi. org/10.21037/tlcr-21-144

Conflicts of Interest: All authors have completed the ICMJE uniform disclosure form (available at http://dx.doi. org/10.21037/tlcr-21-144). Dr. AP has received honoraria or consulting fees from AstraZeneca, Boehringer Ingelheim, Bristol-Myers Squibb, Eli-Lilly, Merck Sharp \& Dohme, Jansenn, Pfizer, Roche, outside the submitted work. The other authors have no conflicts of interest to declare.

Ethical Statement: The authors are accountable for all aspects of the work in ensuring that questions related to the accuracy or integrity of any part of the work are appropriately investigated and resolved. Experiments were performed under a project license (NO.: NFEC-2020-094) granted by institutional ethics board of Nanfang Hospital, Southern Medical University, in compliance with Chinese national or institutional guidelines for the care and use of animals.
Open Access Statement: This is an Open Access article distributed in accordance with the Creative Commons Attribution-NonCommercial-NoDerivs 4.0 International License (CC BY-NC-ND 4.0), which permits the noncommercial replication and distribution of the article with the strict proviso that no changes or edits are made and the original work is properly cited (including links to both the formal publication through the relevant DOI and the license). See: https://creativecommons.org/licenses/by-nc-nd/4.0/.

\section{References}

1. Bray F, Ferlay J, Soerjomataram I, et al. Global cancer statistics 2018: GLOBOCAN estimates of incidence and mortality worldwide for 36 cancers in 185 countries. CA Cancer J Clin 2018;68:394-424.

2. Kim ES. Chemotherapy Resistance in Lung Cancer. Adv Exp Med Biol 2016;893:189-209.

3. Schiller JH, Harrington D, Belani CP, et al. Comparison of four chemotherapy regimens for advanced non-smallcell lung cancer. N Engl J Med 2002;346:92-8.

4. Zheng D, Wang R, Ye T, et al. MET exon 14 skipping defines a unique molecular class of non-small cell lung cancer. Oncotarget 2016;7:41691-702.

5. Ohashi K, Maruvka YE, Michor F, et al. Epidermal growth factor receptor tyrosine kinase inhibitor-resistant disease. J Clin Oncol 2013;31:1070-80.

6. Yuan M, Huang LL, Chen JH, et al. The emerging treatment landscape of targeted therapy in non-small-cell lung cancer. Signal Transduct Target Ther 2019;4:61.

7. Doroshow DB, Sanmamed MF, Hastings K, et al. Immunotherapy in Non-Small Cell Lung Cancer: Facts and Hopes. Clin Cancer Res 2019;25:4592-602.

8. Steven A, Fisher SA, Robinson BW. Immunotherapy for lung cancer. Respirology 2016;21:821-33.

9. Borghaei H, Gettinger S, Vokes EE, et al. Five-Year Outcomes From the Randomized, Phase III Trials CheckMate 017 and 057: Nivolumab Versus Docetaxel in Previously Treated Non-Small-Cell Lung Cancer. J Clin Oncol 2021;39:723-33.

10. Liu Y, Wang H, Deng J, et al. Toxicity of tumor immune checkpoint inhibitors-more attention should be paid. Transl Lung Cancer Res 2019;8:1125-33.

11. Kennedy LB, Salama AKS. A review of cancer immunotherapy toxicity. CA Cancer J Clin 2020;70:86-104.

12. Chen N, Fang W, Zhan J, et al. Upregulation of PDL1 by EGFR Activation Mediates the Immune Escape in EGFR-Driven NSCLC: Implication for Optional Immune 
Targeted Therapy for NSCLC Patients with EGFR Mutation. J Thorac Oncol 2015;10:910-23.

13. Pardoll DM. The blockade of immune checkpoints in cancer immunotherapy. Nat Rev Cancer 2012;12:252-64.

14. Hellmann MD, Rizvi NA, Goldman JW, et al. Nivolumab plus ipilimumab as first-line treatment for advanced nonsmall-cell lung cancer (CheckMate 012): results of an open-label, phase 1, multicohort study. Lancet Oncol 2017;18:31-41.

15. Antonia S, Goldberg SB, Balmanoukian A, et al. Safety and antitumour activity of durvalumab plus tremelimumab in non-small cell lung cancer: a multicentre, phase $1 \mathrm{~b}$ study. Lancet Oncol 2016;17:299-308.

16. Lynch TJ, Bondarenko I, Luft A, et al. Ipilimumab in combination with paclitaxel and carboplatin as first-line treatment in stage IIIB/IV non-small-cell lung cancer: results from a randomized, double-blind, multicenter phase II study. J Clin Oncol 2012;30:2046-54.

17. Nakamura A, Inoue A, Morita S, et al. Phase III study comparing gefitinib monotherapy $(\mathrm{G})$ to combination therapy with gefitinib, carboplatin, and pemetrexed (GCP) for untreated patients (pts) with advanced non-small cell lung cancer (NSCLC) with EGFR mutations (NEJ009). J Clin Oncol 2018;36:abstr 9005.

18. Liang H, Liu X, Wang M. Immunotherapy combined with epidermal growth factor receptor-tyrosine kinase inhibitors in non-small-cell lung cancer treatment. Onco Targets Ther 2018;11:6189-96.

19. Gettinger S, Hellmann MD, Chow LQM, et al. Nivolumab Plus Erlotinib in Patients With EGFR-Mutant Advanced NSCLC. J Thorac Oncol 2018;13:1363-72.

20. Chen Y, Xia R, Huang Y, et al. An immunostimulatory dual-functional nanocarrier that improves cancer immunochemotherapy. Nat Commun 2016;7:13443.

21. Zhang P, Huang Y, Liu H, et al. A PEG-Fmoc conjugate as a nanocarrier for paclitaxel. Biomaterials 2014;35:7146-56.

22. Chen Y, Sun J, Huang Y, et al. Improved Cancer Immunochemotherapy via Optimal Co-delivery of Chemotherapeutic and Immunomodulatory Agents. Mol Pharm 2018;15:5162-73.

Cite this article as: Diao D, Zhai J, Yang J, Wu H, Jiang J, Dong X, Passaro A, Aramini B, Rao S, Cai K. Delivery of gefitinib with an immunostimulatory nanocarrier improves therapeutic efficacy in lung cancer. Transl Lung Cancer Res 2021;10(2):926-935. doi: 10.21037/tlcr-21-144
23. Rebuzzi SE, Alfieri R, La Monica S, et al. Combination of EGFR-TKIs and chemotherapy in advanced EGFR mutated NSCLC: Review of the literature and future perspectives. Crit Rev Oncol Hematol 2020;146:102820.

24. Liu W, Zhang L, Xiu Z, et al. Combination of Immune Checkpoint Inhibitors with Chemotherapy in Lung Cancer. Onco Targets Ther 2020;13:7229-41.

25. García-González J, Ruiz-Bañobre J, Afonso-Afonso FJ, et al. PD-(L)1 Inhibitors in Combination with Chemotherapy as First-Line Treatment for Non-SmallCell Lung Cancer: A Pairwise Meta-Analysis. J Clin Med 2020;9:2093.

26. Gadgeel S, Rodríguez-Abreu D, Speranza G, et al. Updated Analysis From KEYNOTE-189: Pembrolizumab or Placebo Plus Pemetrexed and Platinum for Previously Untreated Metastatic Nonsquamous Non-Small-Cell Lung Cancer. J Clin Oncol 2020;38:1505-17.

27. Pollack BP, Sapkota B, Cartee TV. Epidermal growth factor receptor inhibition augments the expression of MHC class I and II genes. Clin Cancer Res 2011;17:4400-13.

28. Oxnard GR, Yang JC, Yu H, et al. TATTON: a multi-arm, phase Ib trial of osimertinib combined with selumetinib, savolitinib, or durvalumab in EGFR-mutant lung cancer. Ann Oncol 2020;31:507-16.

29. Yang JC, Shepherd FA, Kim DW, et al. Osimertinib Plus Durvalumab versus Osimertinib Monotherapy in EGFR T790M-Positive NSCLC following Previous EGFR TKI Therapy: CAURAL Brief Report. J Thorac Oncol 2019;14:933-9.

30. Holmgaard RB, Zamarin D, Li Y, et al. Tumor-Expressed IDO Recruits and Activates MDSCs in a Treg-Dependent Manner. Cell Rep 2015;13:412-24.

31. Yu J, Du W, Yan F, et al. Myeloid-derived suppressor cells suppress antitumor immune responses through IDO expression and correlate with lymph node metastasis in patients with breast cancer. J Immunol 2013;190:3783-97.

32. Uyttenhove C, Pilotte L, Théate I, et al. Evidence for a tumoral immune resistance mechanism based on tryptophan degradation by indoleamine 2,3-dioxygenase. Nat Med 2003;9:1269-74. 